Address correspondence to: Eline T. Luning Prak, Department of Pathology and Laboratory Medicine, Perelman School of Medicine, University of Pennsylvania, Philadelphia, Pennsylvania 19104, USA. Phone: 215.746.5768; Fax: 215.573.6317; E-mail: luning@mail.med.upenn.edu.

1. Giblett ER, Anderson JE, Cohen F, Pollara B, Meuwissen HJ. Adenosine-deaminase deficiency in two patients with severely impaired cellular immunity. Lancet. 1972;2(7786):1067-1069.

2. Blackburn MR, Kellems RE. Adenosine deaminase deficiency: metabolic basis of immune deficiency and pulmonary inflammation. Adv Immunol. 2005;86:1-41.

3. Carson DA, Kaye J, Seegmiller JE. Lymphospecific toxicity in adenosine deaminase deficiency and purine nucleoside phosphorylase deficiency: possible role of nucleoside kinase(s). Proc Natl Acad Sci US A. 1977;74(12):5677-5681.

4. Thompson LF, et al. Metabolites from apoptotic thymocytes inhibit thymopoiesis in adenosine deaminase-deficient fetal thymic organ cultures. J Clin Invest. 2000;106(9):1149-1157.

5. Apasov SG, Blackburn MR, Kellems RE, Smith PT, Sitkovsky MV. Adenosine deaminase deficiency increases thymic apoptosis and causes defective T cell receptor signaling. J Clin Invest. 2001; 108(1):131-141.

6. Blackburn MR, Datta SK, Kellems RE. Adenosine deaminase-deficient mice generated using a two-stage genetic engineering strategy exhibit a combined immunodeficiency. J Biol Chem. 1998; 273(9):5093-5100.

7. Aldrich MB, Chen W, Blackburn MR, Martinez-Valdez H, Datta SK, Kellems RE. Impaired germinal center maturation in adenosine deaminase deficiency. J Immunol. 2003;171(10):5562-5570.

8. Junger WG. Immune cell regulation by autocrine purinergic signalling. Nat Rev Immunol. 2011; 11(3):201-212.

9. Sauer AV, Aiuti A. New insights into the pathogenesis of adenosine deaminase-severe combined immunodeficiency and progress in gene therapy. Curr Opin Allergy Clin Immunol. 2009;9(6):496-502.

10. Sauer AV, et al. Alterations in the adenosine metabo- lism and CD39/CD73 adenosinergic machinery cause loss of Treg cell function and autoimmunity in ADA-deficient SCID. Blood. 2012;119(6):1428-1439.

11. Cancro MP, D'Cruz DP, Khamashta MA. The role of B lymphocyte stimulator (BLyS) in systemic lupus erythematosus. J Clin Invest. 2009;119(5):1066-1073.

12. Sauer AV, Morbach H, Brigida I, Ng Y-S, Aiuti A, Meffre E. Defective B cell tolerance in adenosine deaminase deficiency is corrected by gene therapy. J Clin Invest. 2012;122(6):2141-2152.

13. Minguet S, Huber M, Rosenkranz L, Schamel WW, Reth M, Brummer T. Adenosine and cAMP are potent inhibitors of the NF-kappa B pathway downstream of immunoreceptors. Eur J Immunol. 2005;35(1):31-41.

14. Meffre E. The establishment of early B cell tolerance in humans: lessons from primary immunodeficiency diseases. Ann NY Acad Sci. 2011;1246:1-10.

15. Nemazee D. Receptor editing in lymphocyte development and central tolerance. Nat Rev Immunol. 2006;6(10):728-740.

16. Morris SC, Cheek RL, Cohen PL, Eisenberg RA. Autoantibodies in chronic graft versus host result from cognate T-B interactions. J Exp Med. 1990; 171(2):503-517.

\title{
Telomere stability and carcinogenesis: an off-again, on-again relationship
}

\author{
Jennifer J. Wanat and F. Brad Johnson
}

Department of Pathology and Laboratory Medicine, University of Pennsylvania School of Medicine, Philadelphia, Pennsylvania, USA.

\begin{abstract}
Previous studies in mice have demonstrated antagonistic effects of telomerase loss on carcinogenesis. Telomere attrition can promote genome instability, thereby stimulating initiation of early-stage cancers, but can also inhibit tumorigenesis by promoting permanent cell growth arrest or death. Human cancers likely develop in cell lineages with low levels of telomerase, leading to telomere losses in early lesions, followed by subsequent activation of telomerase. Mouse models constitutively lacking telomerase have thus not addressed how telomere losses within telomerase-proficient cells have an impact on carcinogenesis. Using a novel transgenic mouse model, Begus-Nahrmann et al. demonstrate in this issue of the JCI that transient telomere dysfunction in telomerase-proficient animals is a potent stimulus of tumor formation.
\end{abstract}

\section{Telomeres and telomerase in cancer}

Telomeres have a mixed reputation when it comes to cancer. On the one hand, the chromosome-protective functions of telomeres (capping) can be lost with the shortening of telomeres that accompanies cell division, which in turn can limit cell proliferation. When telomeres become critically short and uncapped, they lose their ability to disguise the linear ends of chromosomes from the DNA damage and checkpoint

Conflict of interest: The authors have declared that no conflict of interest exists.

Citation for this article: J Clin Invest. 2012; 122(6):1962-1965. doi:10.1172/JCI63979. response machinery, which - depending on cell context - leads to cell-cycle arrest (senescence) or cell death (1). Thus loss of telomere reserves may stymie a clone of incipient cancer cells before it can give rise to a significant tumor. On the other hand, rare cells that have sufficiently inactivated their checkpoint response machinery (e.g., via mutation) may continue to divide despite telomere losses. In the case of cultured human fibroblasts, inactivation of the p53 and p16/Rb pathways enables bypass of senescence (2). Uncapped telomeres are prone to recombination, including ligation to other uncapped telomeres, yielding dicentric chromosomes that, fol- lowing a tug-of-war at mitosis, generate nondisjunction events or internal chromosome breaks. Cycles of these so-called breakage-fusion-bridge events drive gene sequence and copy number changes leading to cell dysfunction and death, which, in human fibroblasts that have bypassed senescence, is called crisis. But they also provide fertile ground from which rare variants can emerge to form tumors (3). Therefore, a question of fundamental importance is whether telomere losses play net inhibitory or stimulatory roles in carcinogenesis. A correlative question of greater practical importance is whether inhibition of the telomere-lengthening enzyme telomerase is likely to benefit cancer patients.

In humans, telomerase activity is under strict control, in part via epigenetic regulation of genes encoding its components, including the TERT catalytic protein and the TERC template RNA (4). Although telomerase can be detected in the progenitor cells of highly proliferative tissues, its activity is nonetheless insufficient for preventing age-related decreases in telomere lengths. Thus, telomeres would be expected to shorten in a runaway premalignant clone of cells. Indeed, premalignant lesions are 
Table 1

Characteristics of HCC tumors

\begin{tabular}{|c|c|c|c|c|c|}
\hline Strain & Critically short telomeres & Chromosome aberrations & $\gamma-H 2 A X$ foci & Microscopic tumor foci & Macroscopic tumors \\
\hline mTerc $+/+$ TTD- & + & + & + & + & ++ \\
\hline mTerc $+/+$ TTD+ & ++ & +++ & + & +++ & +++ \\
\hline G3 $\mathrm{mTerc}^{-1-}$ & +++ & t++ & t++ & ++ & + \\
\hline
\end{tabular}

Comparison of telomerase-proficient mice $\left(m \mathrm{Terc}^{+/+}\right)$subjected to transient telomere dysfunction during tumorigenesis $\left(\mathrm{TTD}^{+}\right)$, TTD ${ }^{-}$controls, and late generation telomerase-deficient mice (G3 $\mathrm{mTerc}^{--}$). The number of plus symbols indicates relative frequencies. Microscopic foci reflect presumptive early lesions. Note that TTD+ and G3 mTerc ${ }^{-/-}$mice each have increased chromosome aberrations and microscopic tumor foci, but ongoing telomere dysfunction and genome-wide DNA breaks in the later strain (indicated by elevated numbers of $\gamma$-H2AX foci) are associated with fewer (and smaller) macroscopic tumors.

characterized by extremely short telomeres, consistent with shortening limiting further cancer progression $(5,6)$. Accordingly, forced telomerase expression immortalizes human cultured primary fibroblasts, pointing to the strong proliferative barriers evoked by uncapped telomeres (7). Similarly, $\mathrm{mTerc}^{-/}$mice, when crossed for several generations to allow telomeres to shorten significantly (e.g., G3), generally have fewer mature tumors, particularly when the p53-dependent checkpoint is intact (8). In contrast, genome instability driven by telomere dysfunction increases the initiation of early-stage cancer lesions. For example, later generation $m \mathrm{Terc}^{-1-}$ mice carrying an $\mathrm{Apc} \mathrm{Min}^{\mathrm{Min}}$ allele develop higher numbers of intestinal microadenomas than $m \mathrm{Terc}^{+/+} A p c^{\mathrm{Min} /{ }^{+}}$or early generation $m \mathrm{Terc}^{-/-} A p c^{\mathrm{Min} /+}$ controls, although ultimately, the late generation mice develop fewer macroadenomas (9). These observations raise the following question: if telomerase were activated following telomere dysfunction, would the telomere dysfunction promote or inhibit carcinogenesis overall? The nearly ubiquitous presence in human cancers of telomere length-maintenance mechanisms (usually telomerase, or sometimes an alternative recombination-based mechanism called ALT) together with the capacity of telomerase inhibition to compromise tumor growth suggest that functional telomeres are critical to cancer progression (10).

\section{Novel mouse models addressing roles for telomeres and telomerase in carcinogenesis}

To address the capacity of telomerase to support carcinogenesis following telomere dysfunction, in this issue of the JCI, BegusNahrmann et al. report on their creation of a mouse carrying a liver-specific doxycyclineinducible (DOX-inducible) transgene encoding a dominant-negative form of TRF2 (11). TRF2 is a component of a protein complex called Shelterin and plays critical roles in telomere capping, in part by preventing the ATM checkpoint kinase from recognizing the telomere as broken DNA (12). A clever feature of this system is that, since transient telomere dysfunction (TTD, i.e., uncapping) can be induced at any time in animals possessing functional telomerase, TTD effects can be addressed at different stages of cancer progression.

When the transgenic mice were treated at 15 days of age with a hepatocellular carcinoma-inducing (HCC-inducing) agent diethylnitrosamine (DEN), followed by treatment with DOX at 2 to 3 months of age to induce TTD prior to the development of tumors, the numbers of microscopic dysplastic foci and fully developed tumors appearing at 6 to 12 months of age were increased compared with those in mice in which telomere capping was maintained. TTD induction also elevated rates of chromosome aberrations, suggesting that higher rates of oncogenic mutations enhanced tumorigenesis. In contrast, DENtreated G3 m $\mathrm{Terc}^{-/-}$mutants developed less numerous and smaller tumors than even the non-DOX induced TTD strain, despite increased numbers of chromosome aberrations and dysplastic foci (Table 1). Therefore, TTD enhances the initiation of HCC cancers, but persistent telomere dysfunction is deleterious to cancer cell survival, and thus telomerase facilitates the development into mature tumors of early lesions that have experienced telomere dysfunction. Furthermore, by inducing TTD in mice with established HCC at 11 to 13 months of age and following tumor growth using MRI, the authors observed increased tumor size in the DOX-treated mice relative to the controls, indicating that TTD can also aid in cancer progression (11).

Curiously, telomere lengths in TTDinduced tumors were shorter than those in tumors from mice in which telomere dysfunction was not induced. The authors suggest that TTD specifically enhances tumor formation in cells with short telomeres. How this short telomere phenotype is maintained in the presence of telomerase is unclear, but it is interesting that modest telomere lengths are often found in telomerase-positive cancers and that there are correlations between chromosome aberrations and short telomeres in human tumors (13), suggesting that short telomeres may convey some advantage to cancer cells.

Findings complementary to those of Begus-Nahrmann et al. have just been published by the DePinho group, which engineered systems for restoring telomerase activity within an $m$ Tert $/$ - background (14, 15). Pten ${ }^{-/-} p 53^{-/-}$mutant mice (naturally possessing telomerase) displayed early prostate cancer lesions by nine weeks of age and developed large and invasive adenocarcinomas by 24 weeks. Although G3/G4 mert $^{-/}$ Pten ${ }^{-/} p 53^{-/-}$mice also showed cancer initiation by nine weeks, few tumors progressed further, and those that did remained small and were accompanied by high levels of apoptosis and DNA damage checkpoint activation compared with telomerase-positive counterparts. Thus, although critical telomere shortening due to telomerase deficiency may aid cancer initiation, progression is hampered by subsequent apoptosis and DNA-damage responses. Importantly, telomerase-deficient G3/G4 mice in which telomerase was restored at the point of cancer initiation developed invasive carcinomas after 24 weeks, similarly to naturally telomerase-proficient mice. Moreover, $25 \%$ of these mice also displayed skeletal metastases, again suggesting that periods of TDD-induced genome instability, followed by telomerase-dependent stabilization, can promote cancer progression (14). Similar results were obtained using $m$ Tertand Atm-deficient mice in which induction of transgenic mTert stimulated T cell lymphomas. Of note, subsequent inactivation of telomerase in the tumors selected for telomere lengthening by ALT, again point- 
ing to the importance of telomere maintenance in mature tumors (15). Together, the findings from the two research groups indicate that transient telomere dysfunction prior to, concomitant with, or following the initiation of cancer can drive tumorigenesis, provided it is supported by subsequent telomere stabilization.

In addition to addressing roles for TTD and telomerase in carcinogenesis, both sets of findings have revealed additional insights. Of particular note, HCCs in mice with TTD had changes in gene expression and chromosome aberrations similar to those observed in human HCCs, including gains in chromosome 15 , which carries the $c-M y c$ locus linked to human liver carcinogenesis (11). Furthermore, prostate tumors emerging from $m$ Tert $^{-/}{ }^{-}$Pten ${ }^{-1-} p 53^{-/-}$mice in which telomerase activity was restored revealed losses in Smad4, encoding a TGF- $\beta$ family member. Remarkably, Pten ${ }^{-/} p 53^{-/-}$ Smad4 $4^{-/}$mice were particularly prone to prostate cancer, including metastases to bone (14). Thus, despite differences in human and mouse telomere biology (see below), the mouse models have proven themselves valuable guides on the path to understanding human cancer.

\section{Implications of the new findings}

The new findings suggest that TTD in cells possessing active telomerase or in whose progeny telomerase can become activated can contribute to cancer progression. Telomerase inhibitors are being actively tested in clinical trials for cancer, and the new findings raise the possibility that short-term telomerase inhibition in mature tumors will do more harm than good, i.e., TTD might stimulate the appearance of new mutant clones, some of which could promote tumor progression. By the same token, the new findings are consistent with evidence that long-term inhibition of telomerase may be of therapeutic benefit. Also of note, inhibition of telomerase may favor the appearance of tumor subclones that use ALT to maintain telomeres, although as described in the next section, ALT probably emerges at lower frequencies in human than in murine premalignant cells. Thus, studies of telomerase inhibitors as potential therapies for human cancer certainly remain important avenues of investigation.

\section{Caveats based on differences between mice and humans}

It is important to note that the new findings might overestimate the importance of TDD in promoting carcinogenesis in humans because of several key differences between mouse and human telomere biology. Telomere lengths of inbred mouse lines are approximately five times those of humans. Secondly, telomerase activity is less restricted in mice (16), and thus cells that have incurred a period of TDD are more likely to be rescued by telomerase in mice than in humans. Finally, although human and murine cells share p53-dependent checkpoint responses to telomere dysfunction, human cells possess additional responses, including a p16/INK4a-dependent checkpoint $(17,18)$. This may help prevent human cells from bypassing checkpoints to adopt telomerase or ALT-based mechanisms of telomere maintenance (which occur at higher frequencies in mice). Considering these factors, it appears that humans may have evolved a system designed to use telomere shortening as a guard against cancer, whereas mice, which generally maintain telomeres in a capped state, respond less robustly when capping is lost. These considerations may in part explain the approximately 10,000-fold higher rates of cancer, corrected for cell divisions and life span, in mice compared with humans and are consistent with the dramatic capacity of forced expression of telomerase to immortalize cultured human fibroblasts at crisis $\left(\sim 10^{7}\right.$-fold stimulation) compared with the modest effect in murine fibroblasts ( 2-fold stimulation) (7). Overall, it seems likely that in human cells, the robust checkpoint responses to telomere dysfunction coupled with controls on telomerase enable telomeres to subserve an anticancer function. Nonetheless, in settings where telomeres are pathologically short, e.g., due to high mucosal cell turnover caused by immune-mediated damage in inflammatory bowel disease or due to telomerase deficiency in dyskeratosis congenita patients (8), the protumorigenic effects of TTD may be magnified. In these conditions, perhaps the large numbers of cells with telomere dysfunction compared with the small number of premalignant cells with short telomeres in normal individuals provide greater opportunity for emergence of tumorigenic cells overall. Additional investigations, including detailed studies of telomere dynamics at different stages of carcinogenesis in human tissues, are needed to evaluate these ideas further.

\section{Open questions}

Several questions are raised by the new sets of findings: might transient inhibition of telomerase in cancer patients be potentially harmful, and will sustained inhibition be required for therapeutic benefit? How significant is the possibility that telomerase inhibition will select for ALT-dependent tumor subclones? Furthermore, at what stages of tumorigenesis does functionally important telomere uncapping occur? Assays designed to address telomere capping (rather than telomere length) will be useful in addressing this question (1, 19, 20). Finally, do the broad age-related declines in telomere lengths in multiple tissues serve to promote carcinogenesis in the elderly? Although telomere shortening in rare cells that are dividing out of control within a young individual may serve to inhibit cancer progression, if most cells within an elderly individual naturally have shortened telomeres, the net effect may be to promote cancer. Answers to these questions will aid in tailoring telomere-related cancer therapies for young and old alike.

\section{Acknowledgments}

We thank J.E. Johnson and M.B. Billmire for comments on the manuscript. This work was supported by a postdoctoral fellowship to J.J. Wanat from the American Federation for Aging Research and NIH grant R01 AG021521 to F.B. Johnson.

Address correspondence to: F. Brad Johnson, Department of Pathology and Laboratory Medicine, University of Pennsylvania School of Medicine, 422 Curie Boulevard, Philadelphia, Pennsylvania 19104, USA. Phone: 215.573.5057; Fax: 215.573.6317; E-mail: johnsonb@mail.med.upenn.edu.

1. d'Adda di Fagagna F, et al. A DNA damage checkpoint response in telomere-initiated senescence. Nature. 2003;426(6963):194-198.

2. Shay JW, Pereira-Smith OM, Wright WE. A role for both RB and p53 in the regulation of human cellular senescence. Exp Cell Res. 1991;196(1):33-39.

3. Artandi SE, et al. Telomere dysfunction promotes non-reciprocal translocations and epithelial cancers in mice. Nature. 2000;406(6796):641-645.

4. Forsyth NR, Wright WE, Shay JW. Telomerase and differentiation in multicellular organisms: turn it off, turn it on, and turn it off again. Differentiation. 2002;69(4-5):188-197.

5. Meeker AK, et al. Telomere shortening is an early somatic DNA alteration in human prostate tumorigenesis. Cancer Res. 2002;62(22):6405-6409.

6. Plentz RR, et al. Telomere shortening and inactivation of cell cycle checkpoints characterize human hepatocarcinogenesis. Hepatology. 2007;45(4):968-976.

7. Wright WE, Shay JW. Telomere dynamics in cancer progression and prevention: fundamental differences in human and mouse telomere biology. Nat Med. 2000;6(8):849-851.

8. Artandi SE, DePinho RA. Telomeres and telomerase in cancer. Carcinogenesis. 2010;31(1):9-18.

9. Rudolph KL, Millard M, Bosenberg MW, DePinho 
RA. Telomere dysfunction and evolution of intestinal carcinoma in mice and humans. Nat Genet. 2001;28(2):155-159.

10. Roth A, Harley CB, Baerlocher GM. Imetelstat (GRN163L)-telomerase-based cancer therapy. Recent Results Cancer Res. 2010;184:221-234.

11. Begus-Nahrmann Y, et al. Transient telomere dysfunction induces chromosomal instability and promotes carcinogenesis. J Clin Invest. 2012; 122(6):2283-2288

12. Karlseder J, Broccoli D, Dai Y, Hardy S, de Lange T. p53- and ATM-dependent apoptosis induced by telomeres lacking TRF2. Science. 1999; 283(5406):1321-1325.

13. Plentz RR, et al. Telomere shortening correlates with increasing aneuploidy of chromosome 8 in human hepatocellular carcinoma. Hepatology. 2005; 42(3):522-526.

14. Ding Z, et al. Telomerase reactivation following telomere dysfunction yields murine prostate tumors with bone metastases. Cell. 2012;148(5):896-907.

15. $\mathrm{Hu}$ J, et al. Antitelomerase therapy provokes ALT and mitochondrial adaptive mechanisms in cancer. Cell. 2012;148(4):651-663.

16. Horikawa I, et al. Differential cis-regulation of human versus mouse TERT gene expression in vivo: identification of a human-specific repressive element. Proc Natl Acad Sci U S A. 2005; 102(51):18437-18442.

17. Khoo CM, Carrasco DR, Bosenberg MW, Paik JH,
Depinho RA. Ink4a/Arf tumor suppressor does not modulate the degenerative conditions or tumor spectrum of the telomerase-deficient mouse. Proc Natl Acad Sci U S A. 2007;104(10):3931-3936.

18. Smogorzewska A, de Lange T. Different telomere damage signaling pathways in human and mouse cells. EMBO J. 2002;21(16):4338-4348.

19. Herbig U, Jobling WA, Chen BP, Chen DJ, Sedivy JM. Telomere shortening triggers senescence of human cells through a pathway involving ATM, p53, and p21(CIP1), but not p16(INK4a). Mol Cell. 2004;14(4):501-513.

20. Takai H, Smogorzewska A, de Lange T. DNA damage foci at dysfunctional telomeres. Curr Biol. 2003;13(17):1549-1556.

\title{
Therapeutic potential of a peptide targeting BCL-2 cell guardians in cancer
}

\author{
Jerry M. Adams \\ Walter and Eliza Hall Institute of Medical Research, Molecular Genetics of Cancer Division, Parkville, Victoria, Australia. University of Melbourne, \\ Department of Medical Biology, Parkville, Victoria, Australia.
}

\begin{abstract}
A promising approach to cancer therapy is to elicit apoptosis with "BH3 mimetic" drugs, which target proteins of the BCL-2 family. As of yet, however, such drugs can target only certain BCL-2 family proteins. Hence, in this issue of the JCI, LaBelle et al. assess instead the therapeutic potential of a "stapled" $\mathrm{BH} 3$ peptide from the BIM protein, which inactivates all its prosurvival relatives. The peptide killed cultured hematologic tumor cells and abated growth of a leukemia xenograft, without perturbing the hematopoietic compartment. Hence, such peptides might eventually provide a new way to treat refractory leukemias.
\end{abstract}

It is increasingly accepted that most, if not all, conventional cytotoxic cancer therapies rely upon eliciting programmed cell death (apoptosis) in the tumor cells, a process regulated principally by the BCL- 2 protein family (1). Interactions among the members of this family serve as a switch determining whether the cell will live or die. In response to intracellular damage, the distant cousins of BCL-2, called BH3-only proteins because they bear only the third of the four $\underline{B} C L-2$ homology domains that characterize this family, are activated and convey the cell death warrant. They use their $\mathrm{BH} 3$ domain to engage and neutralize their prosurvival relatives, e.g., BCL-2, BCL- $\mathrm{x}_{\mathrm{L}}$, and MCL-1, keeping these cell guardians from restraining the family's critical effector proteins, BAX and BAK. Once the guardians have been overcome, certain potent $\mathrm{BH} 3$-only proteins (e.g., BIM) can also directly acti-

Conflict of interest: The author has declared that no conflict of interest exists.

Citation for this article: J Clin Invest. 2012; 122(6):1965-1967. doi:10.1172/JCI64120. vate $\mathrm{BAX}$ and $\mathrm{BAK}$, which then damage the mitochondria and unleash the proteolytic cascade that culminates in cell death (1).

\section{The prospects of $\mathrm{BH} 3$ mimetics}

Oncologists have long dreamed of drugs that would directly flip the apoptotic switch in cancer cells. That dream inspired the development of the first $\mathrm{BH} 3$ mimetic drugs (reviewed in refs. 1,2), the best studied of which are ABT-737 (3) and its orally bioavailable derivative ABT-263 (4). These drugs are specific for certain prosurvival BCL-2 family members; for example, they bind BCL-2 and BCL- $\mathrm{x}_{\mathrm{L}}$ with high affinity but not MCL-1 (Figure 1A). Consequently, as single agents they kill cells whose survival depends primarily on BCL-2 and/or BCL- $\mathrm{x}_{\mathrm{L}}$ but not those containing sufficient MCL-1 to restrain BAX, unless another agent inactivates or eliminates MCL-1 (5). The many promising preclinical findings with ABT-737 and ABT-263 have led to clinical trials of the latter. Notably, with chronic lymphocytic leukemia, which is sustained by high levels of BCL-2, ABT-263 has shown substantial efficacy as a single agent in one-third of patients, even in cases refractory to all conventional therapies and with poor prognostic markers (6).

In vitro and in vivo, ABT-263 and ABT737 can greatly augment the action of diverse conventional chemotherapeutics $(3,4)$, most likely because those agents diminish active MCL-1 levels, but which drug combinations will be tolerable in the clinic remains unclear. For example, the ability of ABT-737 and ABT-263 to engage BCL- $\mathrm{X}_{\mathrm{L}}$, which is the principal guardian of platelet survival (7), provokes a transient thrombocytopenia, and that has proven to be the dose-limiting toxicity for ABT-263 (6). Consequently, a novel BH3-mimetic highly specific for BCL-2 (ABT-199), which has recently entered clinical trial, should permit higher doses and have even greater promise for chronic lymphocytic leukemia and other diseases sustained by BCL-2 (1).

Eventually, BH3 mimetics that effectively target other family members (e.g., MCL-1) are likely to be developed, but the path to such agents is arduous, because as yet no defined organic skeleton adequately mimics the $\alpha$-helix assumed by the 16 - to 26-residue $\mathrm{BH} 3$ domain upon binding to the hydrophobic groove on the surface of its prosurvival relatives. Hence, the development of a new $\mathrm{BH} 3$ mimetic typically commences with screens of some $10^{6}$ chemical building blocks for binding to the BCL-2 family protein target; the resulting weak "hits" must be modified iteratively over 